\title{
Procédés employés pour le recyclage de l'eau en papeterie
}

\author{
PAR
}

\author{
P. Lejeune ET J. Serpaud
}

Ingenieurs à la Société Alsthom, Division Neyruic

Groupe "Techniques des Fluides", Grenoble

\section{1 - Position du problème}

C'est devenu un lieu commun de dire que l'industrie du papier est une grosse consommatrice d'eau. On a aussi tendance à penser qu'il est indispensable qu'elle utilise une eau très pure car on rencontre de nombreuses papeteries le long des rivières et torrents à caractère plus touristique qu'industriel. Les petites papeteries trouvaient dans ces lieux à la fois l'eau et une partie de la force motrice.

Actuellement, les paramètres déterminants dans l'implantation des papeteries ont déjà changé, sauf cas très particuliers, et les effets du recyclage généralisé risquent de modifier totalement le problème.

En France, l'industrie du papier est située au troisième rang des gros consommateurs d'eau.

Les résultats d'une enquête du C.N.P.F. donnaient, pour 1970 , les chiffres suivants, en millions de mètres cubes/jour :

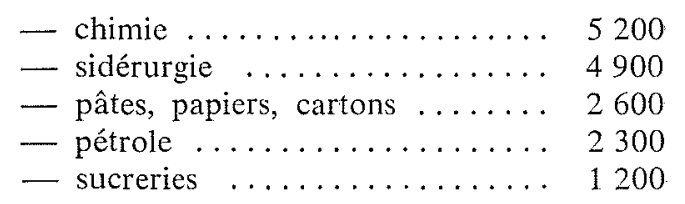

Pour situer ces chiffres, il faut les placer dans le contexte des 33000 millions de mètres cubes par jour d'eau industrielle du VI" Plan.

Sans doute, des statistiques très récentes, établies par les Agences de Bassin, doivent donner des chiffres différents, mais comme nous constatons que toutes les industries soni entrées dans la voie des économies d'eau, les positions respectives peuvent encore changer.

\section{2 - L'eau consommée et l'eau rejetée}

\section{1 - CONSOMMATION}

Elle est ordinairement comptée en $\mathrm{m}^{3}$ par tonnes, avec deux variantes:

- tonnes de pâte produite;

- tonnes de pâte transformée en papier ou carton.

Une usine de pâte à technologie ancienne consomme 150 à $300 \mathrm{~m}^{3} / \mathrm{t}$. Une usine de pâte à technologie moderne se situe aux environs de $50 \mathrm{~m}^{3} / \mathrm{t}$.

Une papeterie à technologie ancienne consomme environ $150 \mathrm{~m}^{3} / \mathrm{t}$ et les pertes de matière sont souvent voisines de $8 \%$. Avec une technologie améliorée, comportant déjà des recyclages internes importants, on se situe environ à 60 ou $80 \mathrm{~m}^{3} / \mathrm{t}$ et on réduit les pertes à 4 ou $5 \%$.

Avec une technologie moderne très étudiée on arrive à 15 ou $20 \mathrm{~m}^{3} / \mathrm{t}$ et on réduit les pertes à 1 ou $2 \%$.

Dans le cas des usines intégrées, les eaux rejetées par la papeterie peuvent souvent être recyclées en fabrique de pâte après récupération des matières en suspension et élimination des impuretés solides. Dans ce cas, la consommation d'eau globale est finalement régie par l'unité de production de cellulose.

Remarque: La consommation proprement dite, c'est-àdire la perte en eau, ou différence entre l'eau prélevée ct l'eau rejetée au milieu naturel, n'est en réalité que de 1 à $2 \mathrm{~m}^{3} / \mathrm{t}$, perdus par évaporation, que ce soit pour la fabrication du papier ou pour la fabrication de la cellulose. 


\section{2 - CIRCUITS}

La consommation est partagée suivant les grands postes suivants :

\subsection{1 - En fabriques de cellulose}

- eaux de cuisson, généralement soumises à évaporation et condensation, après usage, car très chargées en liqueurs, lignine et produits de dégradation de la cellulose;

- eaux de prélavage, le plus souvent concentrées, par lavages à contre-courant et pressages, suivi d'un traite. ment avec les eaux de cuisson;

- eaux de lavage: c'est le débit le plus important; on distingue les eaux de lavage écru (et dans le cas d'un blanchissement au chlore, les eaux de préchloration), lavage sodique et lavage final.

\subsection{2 - En papeteries}

Considérons une machine à table plate classique; on distingue trois circuits: primaire, secondaire et tertiaire, dits : circuits de fabrication.

\section{Circuit primaire}

On réutilise les eaux d'égouttage, prises entre le rouleau de tête et les caisses aspirantes, pour la dilution de la pâte. C'est un circuit court, et presque entièrement équilibré du point de vue hydraulique, avec souvent un léger excédent dû à l'apport d'eau de pulvérisateurs abat-mousse situés au-dessus des surfaces libres. Le temps de réponse de ce circuit est faible. Presque toutes les machines, même anciennes, comportent un tel recyclage qui porte sur des débits d'eau pouvant aller de 100 à $500 \mathrm{~m}^{3}$ par tonne produite.

Ce recyclage en tête de machine permet, par la même occasion, une récupération de la plupart des matières entraînées par les eaux d'égouttage de la pâte sur la table de fabrication.

\section{Circuit SECONDAIRE}

Le circuit est alimenté par les eaux des caisses aspirantes et du cylindre aspirant de toile.
Il peut recevoir également un flux de matières solides provenant des rognures et cassés humides. Les eaux sont dirigées vers les diverses dilutions de la pâte en trituration dans le cas des usines non intégrées.

Avant d'être réutilisées, ces eaux sont, la plupart du temps, traitées sur des récupérateurs de fibres, du type microtamisage pour les rognures et cassés humides, et du type filtre à précouche, filtre à toile, flottation ou décantation, pour les eaux des caisses aspirantes. Ce circuit secondaire est généralement excédentaire en débit, par rapport à la réutilisation, d'une valeur qui équivaut aux apports d'eau claire dans le circuit process, soit de l'ordre de $20 \mathrm{~m}^{3}$ par tonne produite dans le cas d'une usine non intégrée, où la pâte arrive déjà diluée à $40 \mathrm{~g} / \mathrm{l}$ environ à l'entrée des ateliers de production.

Ces valeurs peuvent être supérieures dans le cas de papeteries produisant des papiers ou cartons colorés dans la masse, ou des sortes variées de faible tonnage, nécessitant des lavages fréquents, et ne permettant pas de recycler les eaux secondaires pour la dilution de la pâte en trituration (circuit process partiellement ouvert).

Les pertes de ce circuit sont fortement influencées par le rendement des récupérateurs.

\section{Circuit tertiaire}

On pourrait aussi l'appeler circuit collecteur; on le désigne parfois par : circuit extérieur; il recueille :

- les eaux de rinçage des toiles et des feutres;

- les eaux blanches en excès;

- les refus d'épuration;

- les eaux de pressage;

- les eaux auxiliaires: refroidissements, étanchéité, anneaux liquides des pompes à vide, etc.

Il dépend peu du processus de fabrication. C'est à ce niveau que les économies d'eau les plus importantes peuvent être réalisées, par des utilisations en cascade et des recyclages, afin de réduire au strict minimum les excédents qui devront être envoyés sur la station de traitement aval.

On peut convenir que, sur ces circuits tertiaires, il reste peu ou pas de matières intéressantes à récupérer, si ce n'est l'eau elle-même.
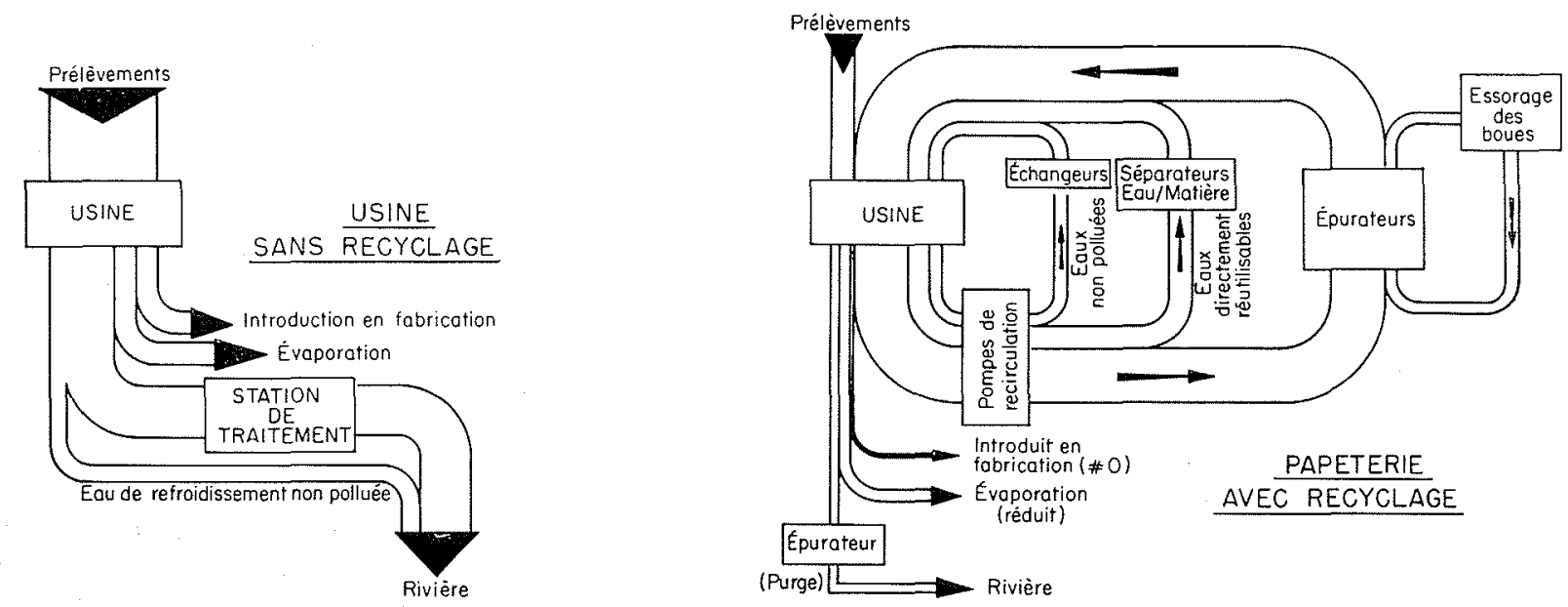

I/ Recyclage. Schéma de circulation d'eau. 


\section{3 - QUALITÉS D'EAU}

Le rapide examen des circuits nous a déjà orienté sur diverses qualités d'eau.

Les circuits auxiliaires utilisent ordinairement des eaux propres car, d'une part les rinçeurs sont sensibles aux bour chages, et d'autre part la fonction rinçage demande une bonne qualité d'eau. Les circuits de refroidissement ont besoin d'eau froide et ont horreur des dépôts sur les parois; les circuits d'étanchéité nécessitent une eau d'où les matières en suspension sont pratiquement absentes.

On pourra cependant faire quelques remarques sur ics qualités d'eau utilisables aux différents postes.

- Un rinçage de toile et un rinçage de feutre sont différents: par exemple le premier se contentera d'une eau véhiculant encore des matières colloïdales, le second les acceptera moins bien.

Dans les deux cas, la pression d'eau a un effet aussi élevé que la quantité d'eau, car il s'agit d'un problème d'énergie.

- L'eau de remplissage et dilution en cuvier n'a aucun besoin d'être très propre, sauf dans le cas de fabrications variées successives, avec changement de teinte ou de composition de la pâte; elle pourra encore contenir des fibres et même des charges, à condition de ne pas recycler les impuretés déjà extraites de la pâte.

Il faudra veiller à ce que cette eau possède un taux de DCO et de DBO pas trop élevé, pour éviter la formation de «slimes» et l'augmentation de teneur en sels favorables à la corrosion, ou pouvant jouer un rôle inhibiteur dans le process normal de fabrication du papier.

\section{3 - Principes applicables dans la recherche d'une optimisation des économies d'eau et de matières}

\section{1 - RECHERCHE DES ÉCONOMIES D'EAU POSSIBLES, AU NIVEAU DES PROCESS DE FABRICATION}

Les process de fabrication peuvent, dans certains cas, être aménagés pour permettre une réduction des consommations d'eau et une diminution des pertes en matières.

En papeterie, on peut citer les améliorations possibles sur le rinçage des feutres par des rinceurs à faible débit, haute pression, travaillant par intermittence, et par des caisses aspirantes de conditionnement, qui entraînent une certainc consommation d'énergie, mais cette dernière est souvent largement compensée par une amélioration du rendement des presses et diminution de l'énergie de séchage du papier.

Au niveau de la sècherie de la machine à papier, l'établissement de circuits vapeur en cascade, avec contrôle efficace du transfert de vapeur et des condensats d'un étage à l'autre, permet une économie de calories et diminue les besoins en eau de refroidissement au niveau du condenseur final.

D'autre part, il convient de citer les diminutions importantes, que l'on peut obtenir, sur les pertes en matières, au niveau de la table de fabrication des machines à papier, par un emploi judicieux d'agents de rétention qui améliorent la fixation des fines et des charges minérales sur les fibres de cellulose. Il en résulte une économie de matières et des rejets moins chargés.

\section{2 - PRATIQUE DE CIRCUITS EN CASCADE PERMETTANT UNE UTILISATION DE LA MEME EAU A PLUSIEURS FINS}

Avant de songer à des recyclages, qui nécessitent souvent des traitements au moins partiels de l'eau avant sa réutilisation, il est intéressant d'observer qu'une même eau peut également servir à plusieurs fins successives, dans un circuit en cascade, lorsque la qualité du rejet à la sortie d'un étage d'utilisation est compatible avec la qualité d'eau requise à l'entrée d'un autre étage.

A titre d'exemple, les eaux de refroidissement d'une machine à papier conviennent bien pour alimenter des rinceurs de feutre, avec l'avantage apporté par une eau légèrement réchauffée. D'autre part, les excédents d'eau de fabrication, après avoir été traités sur des récupérateurs de fibres, peuvent servir successivement au rinçage des toiles de machines et à l'alimentation des anneaux liquides de pompes à vide.

\section{3 - GÉNÉRALISATION DE RECYCLAGES INTERNES, AVEC TRAITEMENTS PARTIELS LOCALISÉS}

Tous les recyclages d'eau admissibles sans traitement spécial étant supposés réalisés, il est généralement encore possible de pousser plus loin les réutilisations d'eau en les soumettant, au préalable, à une épuration partielle, qui peut s'accompagner d'une récupération de matières nobles.

Cette pratique de traitement localisé dans la chaîne de fabrication, avec recyclage immédiat de l'eau et des matières récupérées, est souvent préférable à celle du «tout à l'égoût » avec une énorme station de traitement en aval.

Les appareils de récupération, dont nous parlerons dans lc chapitre suivant, sont particulièrement adaptés à ces traitements partiels de l'eau, dont le but n'est pas d'obtenir une épuration complète, qui permettrait le rejet en milieu naturel, mais une clarification intermédiaire, compatible avec la réutilisation industrielle localisée de cette eau.

Le coût de tels traitements localisés est souvent moins élevé, en investissements comme en frais d'exploitation, que le coût d'un traitement généralisé aval. Il facilite, d'autre part, la récupération sans souillures des matières nobles.

\section{4 - FERMETURE PLUS COMPLETE DES CIRCUITS, PAR RECYCLAGE TOTAL OU PARTIEL DE L'EAU, A LA SORTIE DE LA STATION DE TRAITEMENT AVAL}

Les mesures précédentes ayant été appliquées pour réduire au maximum les consommations d'eau, récupérer les matières nobles, et diminuer le rejet final à l'égout, il reste généralement un certain effluent qu'il convient de traiter sur une station aval, avant retour au milieu naturel.

Dans certaines conditions, les eaux traitées par cette station aval peuvent être, au moins partiellement, recyclées sur l'usine, alors que les boues sont le plus souvent trop polluées ou dégradées pour être récupérables. 
Le maintien d'un effluent minimum reste en général nécessaire pour assurer une déconcentration des circuits en sels dissous, et pour éviter un enrichissement excessif en matières oxydables. Le taux de recyclage admissible est en grande partie conditionné par l'efficacité du traitement aval, dont la qualité, plus ou moins poussée, n'est pas totalement indépendante de son coût.

La tendance actuelle serait, non seulement de limiter au maximum les consommations d'eau à tous les niveaux d'utilisation dans l'usine, mais de pratiquer, chaque fois qu'il est possible, des alimentations en cascade et des recyclages, en opérant à cette fin des traitements partiels localisés. Ceci permet à la fois de réduire les prélèvements d'eau au milieu naturel et limportance de la station de traitement do l'effluent aval.

\section{4 - Quelques exemples de méthodes et appareils utilisés pour permettre de recycler les eaux et récupérer des matières dans la fabrication des papiers et cartons}

\section{1 - OPÉRATIONS DE TAMISAGE}

Des appareils du type tamis sont utilisés en papeterio pour récupérer les matières nobles contenues dans les rognures humides des machines à table plate. Occasionnellement, ces appareils peuvent recevoir la totalité des matic̀res correspondant à la production de la machine, lors d'une casse de la feuille en partie humide.

On utilise à cette fin des tambours épaississeurs tournants, équipés de toiles relativement grossières, des appareils du type trommel, ou des grilles inclinées (grilles courbes), sur lesquelles la pâte humide s'épaissit par écoulement gravitaire.

La nécessité de tirer de ces appareils des capacités de travail importantes, avec un temps de réponse très court, en fait des récupérateurs relativement grossiers.

Les fibres longues sont récupérées avec un bon rendement, mais les fibres courtes et les charges minérales se retrouvent en forte proportion dans les eaux d'égouttage qui restent généralement très riches en matières en suspension, et doivent subir un traitement complémentaire avant de pouvoir être recyclées.

Les matières récupérées, épaissies à 3 à $6 \%$, sont renvoyées directement dans les cuviers de pâte, en tête de machine.

\section{2 - APPAREILS PLUS SPÉCIALEMENT ADAPTÉS AU TRAITEMENT DES EAUX DE FABRICATION, ET A LA RÉCUPÉRATION DES MATIÊRES QU'ELLES CONTIENNENT, EN VUE DE LEUR RECYCLAGE}

Il s'agit, plus spécialement, du traitement des eaux de caisses aspirantes de toile sur les machines à table plate, de l'excédent des eaux de filtration sur les machines à forme ronde, des eaux récupérées à la sortie des tamis épaississeurs et, éventuellement, de la totalité des eaux de rinçage de lá toile de machine.
Ces eaux qui contiennent des matières nobles, dans des proportions pouvant aller de $100 \mathrm{mg}$ à $2 \mathrm{~g} / 1$, sont le plus souvent réutilisables pour la dilution de la pâte au niveau de la trituration (usines non intégrées), pour le conditionnement des toiles de machine, les excédents pouvant servir à d'autres usages, tels que: pulvérisateurs, abat-mousse, anneaux liquides de pompes à vide, etc.

Il est important que les matières nobles contenues dans ces eaux : particules fines de cellulose, charges minérales, pigments divers, soient réintroduites au plus vite dans le circuit de fabrication, surtout lorsqu'il s'agit de machines produisant des sortes variées en couleur ou en composition fibreuse, avec des changements fréquents.

La fréquence des changements de composition justife également un traitement de clarification efficace des eaux recyclées en trituration, pour préparer la pâte de la fabrication suivante.

D'autre part, la réutilisation des eaux clarifiées pour alimenter les rampes de rinçage, destinées au conditionnement des toiles de machines, ne pose aucun problème si la clarification est efficace.

Les appareils de traitement d'eau, destinées à cet usage, sont plus particulièrement des types suivants:

\subsection{1 - Décanteurs statiques cylindro-coniques}

Nous citerons pour mémoire les grands décanteurs cylindro-coniques, utilisés autrefois dans un grand nombre de papeteries, et plus spécialement dans le cas des machines pour papier journal.

Ces appareils opéraient par décantation naturelle simple, avec reprise des boues à la partie inférieure du cône et surverse latérale d'eau clarifiée, généralement rejetée.

Les vitesses de décantation pour obtenir un rendement acceptable, étaient très faibles (coefficients de Hazen de l'ordre de $0,5 \mathrm{~m}^{3} / \mathrm{m}^{2} /$ heure), ces appareils n'étant que très rarement précédés d'un conditionnement physico-chimique pour favoriser la floculation.

Ces appareils ont été progressivement abandonnés pour les raisons suivantes :

- rendement médiocre sous un encombrement élevé;

- long temps de séjour rendant leur emploi impraticable sur des fabrications variées;

- source d'empoisonnement des circuits par développement des micro-organismes au sein de la masse en décantation.

\subsection{2 - Filtres à toiles}

Trois types de filtres sont utilisés principalement:

- filtres simples;

- filtres à plusieurs zones;

- filtres à précouche.

\subsubsection{1 - Filtres simples}

Ce sont des appareils, en général «rotatifs», dont les eaux d'égouttage sont mélangées à la sortie. Ils récupèrent les fibres et une partie des charges minérales par filtration sur une toile fine sans fin, l'égouttage étant facilité dans certains cas (et pour tous les filtres à toiles) par colonne barométrique ou aspiration sous vide.

Le rendement de récupération et de clarification de ce type d'appareil est très variable suivant les conditions d'utilisation. 
Seuls les appareils très largement dimensionnés, travaillant sur des fibres longues et peu chargées, peuvent avoir de bons rendements.

La difficulté d'obtenir une clarification efficace avec un appareil à filtration simple, tient dans le fait que le premier égouttage au travers d'une toile laisse passer une grande partie des éléments fins.

\subsubsection{2 - Filtres A PLUSIEURS ZONES}

Pour remédier au problème précédent, il a été conçu des filtres dont la zone d'égouttage est séparée en plusieurs zones, deux au moins, telles que les eaux de premier égouttage, dites «filtrat trouble», soient séparées des eaux dc deuxième égouttage, dites «filtrat clair».

La «sous-couche» de fibres déposée sur la toile, dans la phase de premier égouttage, sert de médium filtrant pour le deuxième égouttage.

Les filtrats troubles sont la plupart du temps recyclés en tête du filtre.

En vue d'améliorer le rendement de ces appareils, il est conseillé d'injecter de la pâte à fibres longues, dépourvuc de charges minérales, en mélange avec l'effuent à traiter.

Les injections peuvent, dans certains cas, représenter un tonnage dont l'ordre de grandeur approche le tiers de la production de la machine à papier.

On rencontre différents types de construction d'appareils fonctionnant sur ce principe:

- filtres à tambour; à toile incorporée ou à toile sortante (facilite le nettoyage des toiles et l'enlèvement du gâteau);

- filtres à disques (surface de filtration accrue par rapport au volume spécifique).

A condition d'être assez largement dimensionnés, ces appareils ont de bons rendements de récupération, souvent meilleurs que $90 \%$ sur les fibres, et sensiblement inférieurs sur les charges minérales (talc, kaolin, bioxyde de titane, etc.)

La teneur en M.E.S du filtrat clair peut descendre jusqu'aux environs de $10 \mathrm{mg} / \mathrm{l}$ pour des papiers sans charge à base de fibres longues. Elle est de l'ordre de 50 à $100 \mathrm{mg} / \mathrm{l}$ sur des pâtes de vieux papiers non chargées; elle monte aux environs de $100 \mathrm{mg} / 1$ sur des pâtes chargées.

Les filtrats clairs sont, dans la plupart des cas, de qualité convenable pour alimenter des rinceurs de toile équipés de buses appropriées.

Les débits spécifiques de ces appareils sont (en $\mathrm{m}^{3} /$ heure par $\mathrm{m}^{2}$ de surface fiitrante) de l'ordre de :

- 5 à 6 pour papier kraft non chargé;

- 3,5 pour vieux papiers non chargés;

- 1,5 à 2 pour papiers chargés.

\subsubsection{3 - Filtres A PrÉcouche}

Dans le cas du filtre Waco, par exemple, type sans aspiration sous vide, la toile sans fin est garnie en continu sur un système de renvoi extérieur, d'une couche de cellulose formant médium filtrant avant immersion dans la cuve des eaux à filtrer (voir fig. 2).

Cette précouche est constituée préférentiellement de fibres longues, moyennement raffinées, mais on utilise le plus souvent, pour des raisons pratiques, de la pâte prélevée dans les cuviers en tête de machine.

La quantité de précouche est habituellement de 50 à $100 \mathrm{~g} / \mathrm{m}^{2}$, ce qui représente une valeur de l'ordre de $5 \%$

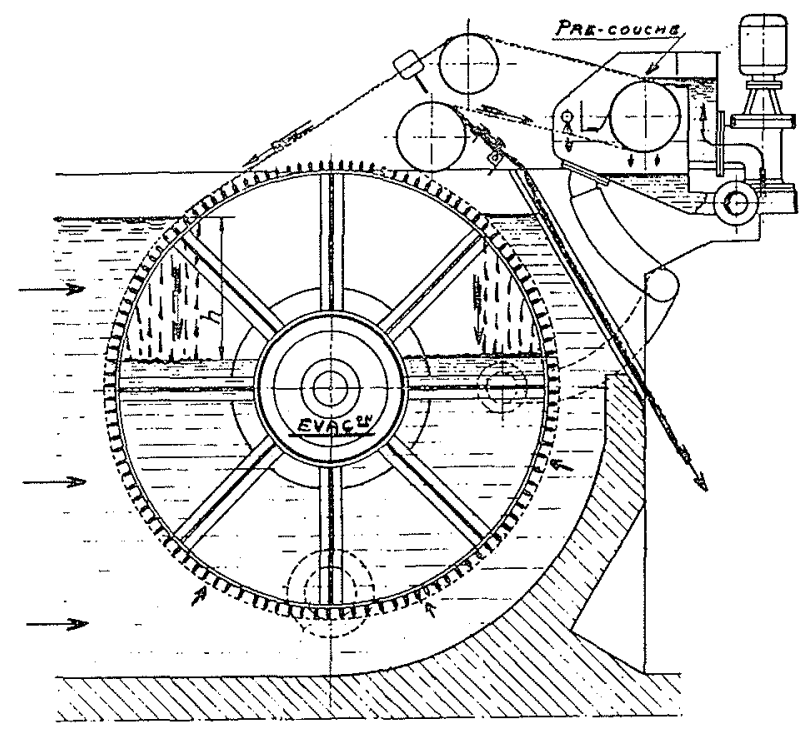

2/ Waco-filtre.

de la production de la machine, et du même ordre de grandeur que la quantité de matière récupérée.

Les débits spécifiques de ces appareils sont de l'ordre do 5 à $15 \mathrm{~m}: 3 /$ heure par mètre carré de surface filtrante.

Le domaine d'application est sensiblement le même que celui des filtres à plusieurs zones et la qualité de l'eau filtrée est comparable.

\subsubsection{4 - Remarque générale}

SUR LES APPAREILS DE FILTRATION

Les divers filtres rotatifs ont pour principal avantage leur faible volume, impliquant un temps de séjour de l'ordre d'une dizaine de minutes; ceci facilite leur intégration dans la chaîne de fabrication, principalement lorsqu'il s'agit d'une machine produisant des sortes variées.

Par contre, le degré de clarification obtenu ne permet que très rarement une qualité d'eau suffisante pour un rejet direct en rivière, car les matières collö̈dales, impliquant une notable proportion de $\mathrm{DCO}$ et $\mathrm{DBO}$, ne sont que très faiblement retenues par ce mode de filtration rapide en surface.

En outre, une difficulté technique se présente lorsque les pâtes sont très engraissées ou lorsqu'elles contiennent beaucoup de matières mucilagineuses, et de charges minérales provoquant une couche colmatante.

\section{3 - RÉCUPÉRATEURS PAR FLOTTATION}

La faible masse spécifique des fibres papetières, et leur propension à retenir les microbulles d'air dans leurs ramifications externes, ont conduit les industriels papetiers à utiliser également des procédés de récupération de matières par flottation, en injectant de l'air dans la veine liquide à traiter.

De nombreux appareils ont vu le jour, travaillant les uns à pression atmosphérique, les autres sous vide. Ils s'apparentent tous plus ou moins aux flottateurs classiques utilisés en traitement d'eau.

Connus de vieille date dans la profession papetière, ces appareils sont passés par des stades d’appréciation divers. 
11 leur était reconnu en effet des qualités très bonnes pour la récupération des fibres raffinées, mais très médiocres pour la récupération des charges, jusqu'à l'apparition sur le marché des polyélectrolytes de synthèse qui ont permis d'améliorer également cette dernière caractéristique.

L'adjonction d'un polyélectrolyte peut permettre, par exemple sur des caux peu chargées en matières minérales, de faire passer le rendement de récupération de 75 à $95 \%$.

Les rendements sont moins bons si les pâtes sont très chargées.

Du point de vue technique, l'air est préalablement dissous dans la totalité de l'effluent à traiter sous une pression de 2 à 3 bars, ou dans une fraction de celui-ci, sous une pression de 4 à 5 bars, mélangée avec le débit total à l'entrée de l'appareil.

Les cellules de flottation sont de formes variées, avec récupération des matières en surface par déversement ou écrémage mécanique.

Dans ce dernier cas, la concentration des matières récupérées peut atteindre 5 à $6 \%$, soit le même ordre de grandeur que celle de la sortie d'un filtre.

Les débits spécifiques sont de l'ordre de $5 \mathrm{~m}^{3}$ /heure par mètre carré pour des appareils de technologie simple; ils peuvent atteindre $10 \mathrm{~m}^{3}$ /heure par mètre carré pour des appareils classiques plus élaborés, et jusqu'à $20 \mathrm{~m}^{3} /$ heure par mètre carré pour des appareils plus compliqués, combinant la flottation avec l'eflet lamellaire des parois inclinées.

La conduite des appareils de flottation sur des fabrications de sortes variées nécessite de porter une attention toute particulière sur la stabilité du $\mathrm{pH}$, de même que sur lo choix et le dosage des réactifs de floculation.

Quand ces appareils sont utilisés et conduits correctement, il est possible d'obtenir des eaux clarifiées contenant de l'ordre de $30 \mathrm{mg} / 1$ de matières en suspension.

De telles eaux peuvent donc être utilisées en recyclage, par exemple pour les rinceurs de toile. Il est certain toutefois, qu'en cas de déréglage, les risques d'afflux de matières dans l'effluent traité sont plus grands que pour un filtre.

\section{4 - UTILISATION PARTICULIËRE DE DÉCANTEURS A LIT DE BOUES, OU LIT FILTRANT DE FLOCONS}

Dans certains cas d'utilisation, lorsque les fabrications sont homogènes, de telle sorte que les matières récupérées puissent être renvoyées en fabrication avec un certain déphasage de temps, il devient possible d'utiliser des décanteurs compacts à lit de boues, dont les vitesses de travail élevées, entre 4 et $7 \mathrm{~m} /$ heure, se rapprochent de celles des appareils à flottation.

Ces appareils présentent une particularité très intéressanto en papeterie, où l'effluent a souvent tendance à floculer naturellement : c'est de fournir par eux-mêmes le lit filtrant de fibres plus ou moins floconneux et d'avoir, sans aucune intervention de produit, un pouvoir de clarification tiès élevé sur les fibres en suspension, de l'ordre de $95 \%$, et surtout, ce qui est très intéressant, un effet d'adsorption remarquable des particules colloïdales.

A titre d'exemple de réalisation l'appareil Seclar, système breveté utilisé dans plusieurs papeteries en France et à l'étranger, est constitué essentiellement comme suit (voir fig. 3) :

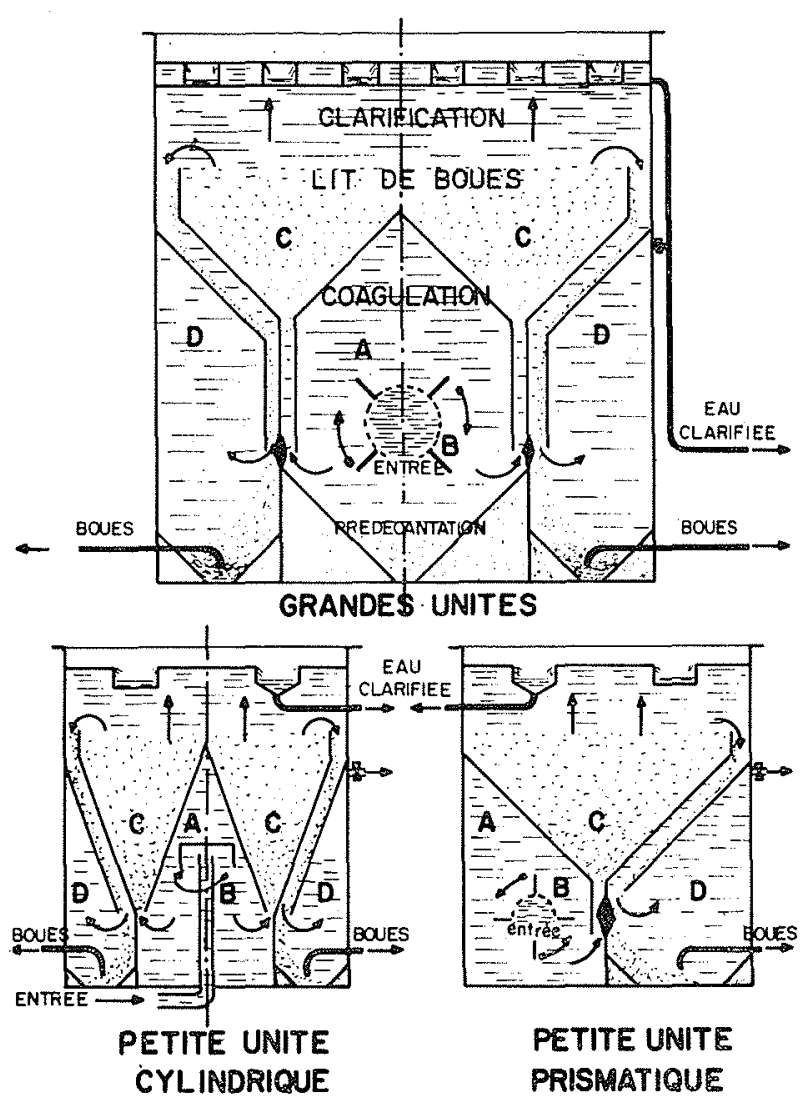

3/ Appareils "Seclar".

- Une chambre de floculation à brassage hydraulique lent, dans laquelle l'efffuent se dépouille progressivement. de ses matières en suspension sous forme de "flocs», de la même façon que lors d'une agitation lente en bécher.

- Une chambre de séparation en forme de dièdre tourné vers le haut, dans laquelle l'effuent, déjà floculé, est introduit le long de l'arête inférieure par une fente de répartition délimitée par une lèvre fixe et une lèvre mobile articulée.

Les flocs décantent dans le dièdre et forment un lit de boues que traverse l'effuent à traiter sous forme d'un contrecourant ascendant, qui assure une recirculation interne permanente de la boue floculée et un grossissement des flocs, ce qui explique les vitesses de décantation élevées obtenues avec ces appareils.

Le "traversement" ascendant du, lit de boues assure, d'autre part, une mise en présence répétée des microparticules et des flocs plus gros qui, par attraction moléculaire, et/ou accrochage mécanique, captent ces éléments fins, à la manière d'une filtration «en profondeur ».

Le niveau de boue se stabilise, à la partie supérieure du dière, sous la forme d'une surface libre de liquide dense, maintenue à niveau constant par un déversoir latéral noyé qui les évacue en continu, par courant de densité, vers les compartiments de décantation secondaire.

L'eau clarifiée primaire se rassemble, au-dessus de la surface libre du lit de boues, et s'évacue par déversement. dans des goulottes de répartition situées à la partie supérieure du décanteur.

Une fraction des eaux, entraînée avec les matières solides, est recueillie à la partie supérieure des chambres de 
décantation secondaire et rejoint, parfaitement clarifiée, les eaux de décantation primaire, provenant des goulottes de répartition.

Les matières récupérées sont rassemblées, par courant de densité, dans des trémies latérales à forte pente, d'où elles sont reprises, par pompe volumétrique ou par gravité, suivant un cycle programmé.

Les trois compartiments, décrits ci-dessus, sont disposés de manière à rencire l'appareil très peu encombrant, avec une parfaite accessibilité de l'ensemble des chambres de travail.

Ces appareils entièrement statiques ont une perte de charge très faible - de l'ordre de quelques décimètres de colonne d'eau - qui permet de les alimenter par gravité, ou de restituer les eaux traitées à un niveau très peu différent du niveau d'alimentation.

II n'est pas cherché, dans les appareils Seclar, une concentration poussée des matières en suspension récupérées, que l'on reprend telles quelles, à concentration de $8-10 \mathrm{~g} / \mathrm{l}$, ou que l'on expédie vers un petit épaississeur extérieur pour porter la concentration à $40 \mathrm{~g} / 1$ environ. Ceci permet d'éviter tout organe tournant à l'intérieur des appareils, et assure un retour plus rapide des matières récupérées, ce qui est également préférable pour éviter le développement des micro-organismes, avantage important dans le cas d'un recyclage.

Le temps de séjour moyen de l'eau, dans les décanteurs Seclar pour eau de papeterie, est de l'ordre de 1 heure. Le temps de séjour moyen des matières décantées est de l'ordre de 1 h 30 à 2 h suivant les concentrations.

Ces nouveaux types de décanteurs ouvrent la voie à des récupérations très poussées des matières solides en suspen.sion : de l'ordre de $95 \%$ sans additifs de floculation, et compris entre 99 et $100 \%$ avec l'appui d'un conditionnement approprié. Ils conviennent aussi bien pour des eaux contenant seulement des matières fibreuses, que pour des mélanges de fibres et de charges minérales, y compris le bioxyde de titane.

Le degré de clarification obtenu par cette méthode est remarquable et très généralement supérieur à ce que l'on peut obtenir par les autres moyens. En particulier, la rétention des pigments, des charges minérales fines, des colles, résines ou autres particules semi-colloïdales, sur les fiocs de cellulose, est plus efficace; ce qui laisse également entrevoir la possibilité de recycler de telles eaux pour des lavages de feutres ou pour d'autres usages en papeterie.

A titre d'exemple, une papeterie française équipée d'un décanteur de ce type, qui traite toutes les eaux rejetées par l'usine, a réussi l'expérience de tourner durant près d'unc semaine en circuit quasi fermé, sans entraver sa production, et avec récupération totale des matières. Il convient de préciser que la décantation des matières en suspension était effectuée cependant sans aucun additif, et que l'usine fabrique elle-même une partie de sa pâte (pâte de paille à la chaux).

De telles opérations, qui conduisent jusqu'à un recyclage quasi total des eaux, ne peuvent toutefois être pratiquées d'une façon définitive qu'à condition de prendre toutes les précautions voulues pour éviter un enrichissement excessif des eaux recyclées en matières oxydables et/ou en matières dissoutes.

Pour cette raison, et on touche ici également au traitement des eaux de circuits auxiliaires (lavages de feutres, vidanges de cuves diverses, effuents provenant de la prépa- ration de sauces de couchage, etc.), il est généralement nécessaire de prévoir en aval une véritable épuration des eaux excédentaires, après laquelle certains recyclages redeviennent à nouveau possibles.

Les traitements complémentaires font appel aux techniques classiques de floculation.

\section{5 - Epuration aval}

\section{1 - CYCLE DE今 MATIÈRES}

Nous avons vu dans les chapitres précédents les différentes possibilités de récupération des matières nobles et comment, grâce à divers appareils de séparation, elles prenaient un chemin court pour revenir à la fabrication.

Les matières dégradées passent par le chemin des circuits auxiliaires et se retrouvent donc dans les égouts.

Le traitement d'épuration aval comprend un premier étage du type physico-chimique, qui va les séparer en grande partie de l'eau sous forme de boues.

$\mathrm{Si}$ on considère ces matières comme totalement dégradées, elles sont justiciables, après mise à l'état «pelletable», de l'abandon sur dépôt spécialisé et autorisé, de l'incinération ou de la transformation en compost.

Par contre, il existe des cas où elles sont encore réutilisables, soit sur des machines produisant des sortes spécialisées de seconde qualité, soit comme couche intérieure do cartons multicouches, soit comme complément dans unc machine utilisant des vieux papiers.

\section{2 - CYCLE DE L'EAU}

On peut affirmer que ]'eau est toujours récupérable, mais il faut penser au prix de cette récupération.

Il faut donc porter son choix sur des appareils assez. simples et d'une grande efficacité. On vise en effet à réduire d'une manière considérable le taux de matières en suspension de toutes sortes (MES) et, d'une manière très forte, le taux des matières oxydables (MOX) donc la $\mathrm{DCO}$ et la $\mathrm{DBO}$.

Dans certains cas d'usines non intégrées, n'utilisant que des pâtes nobles de cellulose, une notable partie de l'eau est recyclable après un traitement physico-chimique constitué par une floculation très soignée, suivie d'une séparation à très haut degré d'efficacité.

Dans ce cas, le rendement sur les MES est de 98-99\% et celui sur les MOX, assez variable, suivant les types d'appareils et le mode de traitement, 20 à $60 \%$.

Dans les cas d'usines travaillant d'autres matières telles que : paille, vieux papiers, ou possédant une petite unité de production de pâte, le problème est plus sérieux car, d'unc part le taux de matières oxydables est élevé et, d'autre part, le rendement sur les MOX est insuffisant, même avec forte purge de déconcentration et en tenant compte de celle qui est faite par le papier produit.

La station d'épuration comprend alors un étage physicochimique suivi d'un étage d'oxydation biologique. La combinaison de ces deux étages et leurs ampleurs respectives donnent lieu à des réalisations très variées.

Il est certain que le travail d'ensemble d'une station d'épuration, reproduisant sur une surface assez réduite le 
travail d'autoépuration de la rivière, place à proximité du lieu d'emploi un débit d'eau apte à être repris pour les fabrications.

Les appareils utilisés pour le traitement aval de l'effuent final sont à l'heure actuelle presque exclusivement des décanteurs.

On rencontre encore un certain nombre de papeteries équipées de bassins de décantation plus ou moins rudimentaires dont l'efficacité est rarement suffisante.

En effet, ces installations très simples posent des problè-. mes pour l'extraction régulière des boues et ne bénéficient pas, la plupart du temps, d'un conditionnement de floculation adéquat à l'entrée pour assurer une bonne sécurité de fonctionnement.

Quel que soit le type de décanteur utilisé, il est certain en effet qu'à ce stade, un conditionnement physico-chimique de floculation, avec réglage du pH et addition d'électrolytes minéraux et/ou organiques pour déstabiliser les suspensions colloïdales, s'avère absolument nécessaire.

Les décanteurs perfectionnés, utilisés le plus souvent a ce stade, sont les décanteurs circulaires raclés qui permettent une évacuation permanente des boues.

Ces appareils existent en plusieurs variantes avec chambre de floculation séparée ou incorporée, épaississeur de boues intégré ou non, certains étant équipés également d'un système de recirculation des boues par hydro-éjecteur en vue d'améliorer les conditions de floculation.

Le coefficient de Hazen de ces décanteurs est compris entre 0,6 et $1,8 \mathrm{~m}^{3} / \mathrm{m}^{2} / \mathrm{h}$; les appareils à recirculation do boues ayant les coefficients les plus élevés.

Dans ce domaine, les appareils à lit de boues du type Seclar sont aussi particulièrement bien placés, en raison de la grande efficacité du lit de boues grâce à la fixation par adsorption des matières colloïdales.

Il en résulte, sur des eaux de papeteries ou de fabrique de cellulose, une réduction très intéressante des matières oxydables et de la couleur des effluents traités.

De nombreuses mesures, sur de grands appareils en fonctionnement et sur des appareils pilotes, ont permis de vérifier que, dans la plupart des cas, l'élimination des matières en suspension se situe aux environs de $99 \%$ et la réduction des matières oxydables atteint ou dépasse même $50 \%$.

Un autre intérêt de ces appareils réside dans leur faible encombrement, leur coefficient de Hazen élevé: 4 à $7 \mathrm{~m}^{3} / \mathrm{m}^{2} / \mathrm{h}$ et leurs possibilités de réalisation modulaire, permettant une extension ultérieure des installations. La qualité des eaux clarifiées permet dans bien des cas, d'envisager leur recyclage.

\section{6 - Conclusion}

Les industriels disposent actuellement des appareils et méthodes capables de permettre des recyclages à taux élevé de l'eau et des matières.

Des difficultés, toutefois, doivent être surmontées pour permettre une extension des recyclages conduisant vers des circuits de plus en plus fermés: augmentation de salinité de l'eau, saturation en matières oxydables, doivent être contrôlées de très près pour éviter des difficultés de fabrication telles que :

- affaiblissement de la résistance humide;

- diminution de la blancheur;

- corrosions dans les circuits;

- développement des micro-organismes, etc.

En conséquence, de tels aménagements doivent êtro conduits pas à pas, en entente étroite avec les exploitants, les spécialistes de traitement des eaux et les conseils en technique papetière.

De plus en plus, le traitement des eaux devra participer au process industriel et il parait normal que les appareils d'épuration se trouvent intégrés aux chaînes de fabrication à l'emplacement le mieux adapté.

En conséquence, il est vraisemblable que les Agences Financières de Bassins considéreront comme de plus en plus normal de participer au financement de telles installations, même si l'industriel concerné devait à long terme y trouver un certain profit, grâce à la récupération de certaines matières nobles.

\section{7 - Bibliographie}

Colas (R.). — «L'eau et la vie». Ed. Prodec (1971).

Col.AS (R.). - «La pollution des eaux». P.U.F., nº 983 (1968)

Maurin (Y.) et DE LA CERda (I. M.). - Réduction de la pollution en papeterie par optimisation des circuits. Revue $A T I P$, vol. 28 (1974)

Lejeune (P.). - Circuits de tête, étude et réalisation. Revue $A T I P$ vol. 24, $\mathrm{n}^{\circ} 4$ (1970).

Bachto (A.). - Rôle de l'industrie chimique dans la lutte contre la pollution des eaux papetières. Revue $A T I P$, vol. $28, \mathrm{n}^{\circ}$ : (1974)

Mörgel (B.). - Les possibilités d'épuration des eaux recyclées ou résiduaires des papeteries compte tenu des procédés chimicophysiques. Revue ATIP, vol. 28, n* 5 (1974).

Berwart (G.). - Epuration des eaux résiduaires à la Cellulose des Ardennes. La Technique de l'eau (novembre 1974).

Lejeune (P.) el Serpaud (J.). - Le procédé Seclar et ses applications en papeterie. Revue ATIP, vol. 28, $\mathrm{n}^{\circ} 5$ (1974).

Brecht (W), Dalpke (H. H), Borner (F.) — "Geschlosseno Wasserkreislauife in weiteren alt-papierwerarbeitenden Papierfabriken » (Fermeture du circuit d'eau de fabrication dans les papeteries utilisant du vieux papier). Trad. C.T.P. 8453 (1974)

Delachanal (M) et Lejeune (P.) - Exemples d'application du décanteur Seclar en traitement d'effuents de l'industrie papetière. Revue La Papeterie, $n^{\circ} 6$ (juin 1974).

TESARTK (I.). - Contribution à la similitude mécanique des couches fluidisées. $15^{\mathrm{e}}$ Congrès AIRH, Istambul (1973)

Edeline (F.), Tesarik (I.) et Vostrcil (J.). - Fluidization of flocs produced in chemical or biological treatment plants. Advances in water pollution research ». Pergamon Press, Londres (1969).

REboux (P.). - «Phénomènes de fluidisation 》. Association Française de Fluidisation (1954)

Serpaud (J.). - Procédés de décantation: les appareils Seclar. Colloque Institut Pastetir, Lyon (décembre 1972).

Benaim (R.), Bergougnou (M.) et Elmaleh (S.). - «Application des lits fluidisés à l'épuration des effluents gazeux et liquides ». S.T.A.P., Grenoble (1971).

Tesarik (I.). - Sédimentation et fluidisation des couches de matériaux granuleux et des flocons dans l'eau, $14^{\circ}$ Congrès $A I R H$ Paris (1971) 
O'Melia (Ch.-R.). - A review of the coagulation process. Public Works (1969)

Hurier (B.). - Evolution des recherches en France dans le domaine du traitcment des eaux résiduaires de fabrique de pâte. Revue Institut Pastew, Lyon (1967-1968).

La lutte contre la pollution de l'eau dans l'industric des pâtes et des papiers. Revue technique industrielle de l'eau, $\mathrm{n}^{\circ} 1$ (1974).

Lekander (K. E.), Albertson (U.), Sundberg (T.). - Conférences de JönKöping: Divers sujets a/s. Traitement des effluents de papeteries; K6 (août-sept. 1972).

De Bouille (C.), Bourdelot (J.-C.), Vilars (J.), Peyron, Serpaud (J.). - Pollution par les effluents de papeteries et traitements. Colloque du S.T.A.P. de Grenoble (novembre 1971).
Belugou (S.). - Le papier malade de ses déchets. Usine Nouvelle (1973).

Hoover (W. E.), Peoples (R. F.) et Hoerner (J. A.). - Waste-water treatment for an integrated pulp and paper mill. Jal W.P.C.F. (mars 1973).

"Contrat de branche » des industries preductives de pâtes, papiers et cartons. Convention du 12 juillet 1972.

Porcile (F.). - Paper and board output up only $2 \%$ (statistiques), Pulp and Paper International (1972).

Berry (M.) et VaroquauX (W.). - La lutte contre la pollution dans les usines de pâte à papier. Annales des Mines (mars 1970).

Henri-Robert (P.). - Usines de pâtes à papier: l'épuration des eaux résiduaires. Nuisances et Environnement (juin 1971).

\section{Abstract}

\section{Water recycling in the papermaking industry}

Paper mills discharge two distinct types of effuent: water containing cellulose waste and water from the machines. It is for the latter, which contains relatively little oxidizable matter, that recycling probiems arise. A further distinction must also be made between mills using fresh (frequently imported) pulp and mills using waste paper stock.

Water quarity requirements vary considerably according to use, e.g. in stuff chests, for wire-cleaning, in boilers, and between production and auxiliary circuits.

Water economy is primarily associated with minimal loss of solids and should therefore be sought first of all in the actual manufacuring process. Substantial economy can be achieved by use of circuits operating "in cascade" at gradually decreas. ing rates of flow. Intexnal recycling with local partial treatment is already in use, but should be more generally applied (sei Fig. 1).

Thus, the effuent is either processed as a whole, or cach of its components by a different method using different equipment. Flocculation can be resorted to in various pro. cesses, e.g. multi-plate, simple or floc-bed sediment traps. Pre-coat filters, flotation equipment and rapid-action sand filters are also suitable.

The following examples of high-performance equipment for present or future use are discussed;

-Waco pre-coat filters (Fig. 3);

- Seclar Hoc-bed separators (Fig. 2).
Recycling by a single station at which all the effuent is processed requires high-performance equipment as regards tola! suspended matter, COD, BOD and colour.

Optimalization studies of circuits and processes are almost invariably necessary for efficient recycling system design. Such studies are carricd out stagewise in close collaboration with the factory management and independent water processing and papermaking specialists.

Contact must also be maintained with the regional finance authorities for all matters relating to cost and pollution.

The following are typical design factors:-

a) Individual equipment: Reduction of oxidizable and suspended matter and colour, buffer effect, discharge, local arrangements, cost, reagent and power requirements, maintenance and supervision, down-times, etc.

b) The overall plant: Modification of sewers, circuits, head losses and water-hammer, COD and BOD control in closedcircuit operation, fermentation, smells, slimes, corrosion, tempcrature rise limits, combination with higher production rates, etc.

c) Non-technical factors: Modification of end products, marketing variations.

Final conclusion: Efficient recycling depends very closely on efficient clarifier operation combined with sound overall design. 